\section{As biografias como um dos caminhos na construção da identidade do profissional da enfermagem}

\section{Biographies as a possible path in the construction of a professional identity in the field of nursing}

Maria Itayra Padilha

Professora-associada do Departamento de Enfermagem e do Programa de Pós-graduação em Enfermagem/Universidade Federal de Santa Catarina (UFSC); pesquisadora do CNPq. Rodovia Amaro Antônio Vieira, 2371/818/bl.A 88034-102 - Florianópolis - SC - Brasil padilha@nfr.ufsc.br

\section{Sioban Nelson}

Diretora e professora da Lawrence S. Bloomberg Faculty of Nursing/University of Toronto. 155 College Street, Rm. 130 M5T 1 P8 - Toronto - ON - Canada

sioban.nelson@utoronto.ca

\section{Miriam Susskind Borenstein}

Professora-associada do Departamento de Enfermagem/ UFSC; pesquisadora do CNPq. Rua Professor Hermínio Jacques, 54/502 88015-180 - Florianópolis - SC - Brasil miriam@nfr.ufsc.br

Recebido para publicação em julho de 2010. Aprovado para publicação em maio de 2011.
PADILHA, Maria Itayra; NELSON, Sioban; BORENSTEIN, Miriam Susskind. As biografias como um dos caminhos na construção da identidade do profissional da enfermagem. História, Ciências, Saúde - Manguinhos, Rio de Janeiro, v.18, supl.1, dez. 2011, p.241-252.

\section{Resumo}

Analisa a importância das biografias na constituição da identidade do profissional de enfermagem, valorizando sua utilização como ferramenta de ensino da história dessa disciplina nos cursos de graduação e pós-graduação. Apresenta um levantamento de biografias de mulheres que contribuíram para o desenvolvimento da enfermagem e cujo legado vem inspirando profissionais dessa área. Conclui que o uso de biografias na educação desses profissionais pode subsidiar a construção de suas identidades, por transmitir aos educandos alguns dos valores da profissão.

Palavras-chave: biografias; identidade profissional; historiografia da enfermagem;

educação profissional; enfermagem.

\section{Abstract}

The article analyzes the role of biographies in constructing a professional identity in the field of nursing and their use as a teaching tool in the history of the field at both undergraduate and graduate nursing courses. It surveys some biographies of women who contributed to the development of the field and who left a legacy that has inspired its providers. The conclusion is that biographies can play a role in nursing education, contributing to the construction of a professional identity by conveying the field's values.

Keywords: biographies; professional identity; historiography of nursing; professional education; nursing. 
$\mathrm{A}$ partir da década de 1960 uma série de estudos realizados especialmente por historiadores e sociólogos priorizou a investigação das interações cotidianas entre atores sociais e as construções resultantes dessas interações. Dessa nova perspectiva, passou-se a afirmar que os indivíduos constroem suas identidades, cuja manutenção depende basicamente das interações mantidas por eles no processo de compreensão de si próprios e de sua relação com a realidade (Eder, 2003; Strath, 2002). Identidades coletivas passaram a ser compreendidas a partir não só de um agregado de interações sociais, mas também da razão políticoestratégica de atores sociais. Nestas últimas duas décadas pode-se considerar que pertence ao senso comum a ideia de que identidades coletivas são construções políticas e sociais e que assim devem ser tratadas. (Hobsbawn, Ranger, 1992; Le Goff, 1990; Burke, 1991). ${ }^{1}$

Trazendo essa reflexão para o campo da enfermagem, entendemos que nossa identidade profissional foi e vem sendo construída a partir de vários aspectos, entre eles a relação da área com a sociedade em termos de seu papel assistencial, educativo, científico, social e político.

Este texto completa a tríade de artigos sobre a importância da história da enfermagem, tendo o primeiro direcionado o olhar para a compreensão do legado deixado pelas personagens que influenciaram e vêm influenciando as práticas de cuidado, a pesquisa e a educação em enfermagem, tomando como foco os conteúdos relativos ao tema nos currículos dos cursos de graduação e de pós-graduação em enfermagem (Padilha, Nelson, 2009). No segundo texto, foi desenvolvida discussão sobre a importância das biografias na construção da identidade profissional da enfermagem, entendendo que a construção política dessa identidade coletiva vem sendo moldada também pelo papel das enfermeiras ${ }^{2}$ nas entidades de classe, nas escolas de formação profissional e na realização de eventos, que têm tido participação cada vez maior, bem como na socialização do conhecimento por meio dos periódicos específicos do setor (Padilha, Nelson, 2011).

Este terceiro texto articula os dois anteriores e analisa a importância das biografias como um dos aspectos constituintes da identidade profissional de enfermagem, valorizando seu uso como ferramenta de ensino da história dessa profissão nos cursos de graduação e pós-graduação. Para atingir esse objetivo é apresentada a biografia de algumas mulheres que contribuíram para o desenvolvimento profissional da enfermagem, cujo legado vem servindo de inspiração para enfermeiras ao longo do tempo.

\section{Conceitos norteadores}

A elaboração deste texto tomou por base especialmente artigos relativos à história da enfermagem no período de 1982 a 2007, constituindo-se no que os historiadores denominam história do presente (Certeau, 1982; Burke, 1991). ${ }^{3}$ Essa relação de passado e presente se estabelece na busca do conhecimento, de modo a pensar o passado por meio de questões que constituem 'o aqui e o agora'. Esse processo envolve a ideia de que as verdades são relativas e estão impregnadas da ideologia daquele que produziu determinado conhecimento.

Pensar e escrever a história do presente permite compreender o contexto histórico de desenvolvimento da enfermagem enquanto profissão, especialmente do ponto de vista 
que nos interessa, que é refletir sobre os modos como a identidade profissional é tratada pelos diversos autores ou enfatizar a importância da história da enfermagem para compreensão da área e da construção identitária individual e coletiva.

Começamos nossa investigação por textos que tratam do ensino da história da enfermagem nos cursos brasileiros de graduação e pós-graduação devido a nosso interesse pelo tema e com base na premissa da importância desse ensino na formação profissional do enfermeiro. Mediante referências obtidas nos primeiros textos, chegamos à segunda fonte de dados, artigos sobre temas relativos à história das mulheres (gênero, biografias) e à prática profissional de enfermagem (currículo, assistência hospitalar e comunitária, organizações de classe, especialidades etc.). Finalmente, uma terceira e última estratégia de busca empregada foi a identificação de textos que tivessem como ponto central a identidade profissional, independente de terem sido ou não escritos por autores da área.

Além da busca das referências nos textos relacionados, pesquisamos artigos sobre assuntos correlatos nas bases de dados Pubmed e Scielo, sem restrição à época de publicação, mas que estivessem disponíveis para cópia online. Foram realizadas buscas também na biblioteca digital da Universidade de Toronto, Canadá. Nessas bases de dados, utilizamos as palavraschave: gênero e história da enfermagem, biografia de enfermeiras, identidade profissional em enfermagem e identidade. Selecionamos para leitura 82 textos, em inglês e português, qualificados como relacionados direta ou indiretamente à análise da identidade profissional da enfermagem. Cabe ressaltar que nem todas as publicações foram escritas por enfermeiras ou relacionadas exclusivamente à enfermagem, em especial aquelas que discutem a identidade profissional (Eisenstadt, 1998; Santos, 1998; Bock, 1999; Oliveira, 2000; Ludke, Boing, 2004; Corsini, 2006). À exceção dos psicólogos que abordam especificamente a identidade profissional na psicologia como algo ainda em construção, sem definição concreta de seu papel social, os demais refletem sobre a identidade profissional em termos conceituais, relacionando a memória individual e coletiva com a noção de profissão (Bock, 1999; Pereira, Pereira Neto, 2003).

\section{Biografias e identidade do profissional de enfermagem}

As biografias ou trajetórias de vida das enfermeiras têm sido utilizadas na educação de enfermagem com várias finalidades - ressaltar o desenvolvimento de uma área profissional específica; orientar a criação de escolas de enfermagem e associações profissionais; criar teorias de enfermagem; investigar os avanços sociais e políticos da profissão. Destaca-se, entretanto, seu uso na contextualização de uma época relacionada ao desenvolvimento da enfermagem enquanto profissão do cuidado, no contexto da disciplina de história da enfermagem, obrigatória em todos os cursos brasileiros de graduação em enfermagem, o que difere da prática em outros países como EUA e Canadá.

As enfermeiras historiadoras cujas obras pesquisamos (Barreira et al., 1997; Kirby, 1998; Lait, 2000) ${ }^{4}$ utilizam o recurso do estudo biográfico como uma das estratégias para refletir com estudantes sobre a profissão e a importância da história da enfermagem para a sua construção identitária (Padilha, 2006). 
No levantamento que realizamos na base de dados Pubmed referente aos últimos 25 anos encontramos várias biografias de mulheres e homens, em países como os EUA, Canadá, Inglaterra, França, Alemanha, Austrália, Índia, Coreia, Japão, Brasil, Espanha e Peru. As biografias revelam que ao longo de sua história essas pessoas tiveram papel essencial no desenvolvimento profissional da enfermagem. Para a obtenção de todas as biografias publicadas sobre enfermeiras ou enfermeiros brasileiros optamos por ampliar essa busca até 2011 e incluímos também a base de dados Scielo e o Google Brasil, considerando que nem todos os periódicos de enfermagem estão cadastrados nas demais bases.

Nessa busca instigante, foi surpreendente identificar cerca de 250 biografias ou trajetórias parciais de vida de profissionais de enfermagem que deixaram marca na história de uma escola, um hospital, uma associação profissional ou comunidade. Dessas, 51 referem-se a Virginia Henderson (EUA); 14 a Lillian Wald (EUA); 13 a Lavinia Dock (EUA); e 23 a enfermeiros brasileiros: Edith M. Fraenckel (três), Haydée G. Dourado (duas), Glete de Alcântara (duas), Zaíra Cintra Vidal (duas), Waleska Paixão (duas), irmã Tereza Notarnicola, Clarice Della Torre Ferrarini, Rachel Haddock Lobo, Maria A. Minzoni, Anna Nery, Anna Nava, irmã Mathilde de Nina, Wanda de Aguiar Horta, Maria Rosa Sousa Pinheiro, Olga Verderese, Hilda Anna Krisch, Grasiela Barroso, Eloita Pereira Neves, Ottilie Hammes, Alice Michaud, Flora Costa Marques, Wilson Kraemer de Paula e Liselotte Hoeschl Ornellas. Nessa seleção incluem-se tanto as biografias completas quanto aquelas que apenas esboçam o perfil biográfico e as escritas in memoriam. O critério seletivo foi a ocorrência do nome da pessoa no título do trabalho, o que pode ter ocasionado algumas omissões.

Florence Nightingale, precursora da enfermagem moderna em todo o mundo, não foi incluída nesses números porque foram encontrados cerca de 350 artigos relacionados à história de sua vida, suas atividades na Guerra da Crimeia e seu desempenho como fundadora, em 1860, da primeira escola de enfermagem moderna, no Hospital Saint Thomas, em Londres.

Quem são essas mulheres e o que elas têm em comum?

As enfermeiras americanas citadas são reconhecidas entre outros aspectos pela atuação significativa junto ao movimento feminista na luta por direitos igualitários. Das três, Virginia Henderson (1897-1996) talvez seja, de todas, a personalidade de maior reconhecimento internacional pois, dos 51 textos a seu respeito - biografias, teorias, entrevistas, legado -, 19 foram publicados em idiomas diferentes do inglês, como japonês, italiano, espanhol, francês e norueguês. Dez matérias foram publicadas em 1996, ano de sua morte, demonstrando essas homenagens sua importância para a enfermagem mundial. O primeiro desses artigos, "Miss Virginia Henderson and I", de Masu Yumaki, foi publicado em 1976 no periódico japonês Kango. O último, de 2007, "Nursing care according to Virginia Henderson in the at home care field", é assinado por Coll et al. (2007) e foi divulgado na Revista de Enfermería, de Barcelona. Um dos motivos que a tornaram famosa e reconhecida mundialmente como teórica de enfermagem foi a edição de seu livro publicado em vários idiomas, The principles and practice of nursing (White, 1978; Yumaki, 1976).

Lillian Wald (1867-1940) criou a American Community Nursing, em 1899, que depois se transformaria na mais importante associação de enfermagem mundial, denominada International Council of Nursing. Encontramos o primeiro artigo publicado a seu respeito 
em 1968, "Try to remember our heritage", e o último em 2009, "Remembering Lillian Wald", ambos em periódicos americanos. Apesar de escritos em diferentes períodos históricos, conclamam o público a preservar a memória de Lillian Wald como ícone nas lutas profissionais das enfermeiras americanas. Mais uma vez a década de 1990 é privilegiada quanto ao número de textos, seis sobre essa personagem (Halamamdaris, 2009; Hawkins, Watson, 2003; Popiel, 1968).

Lavinia Dock (1858-1956) - enfermeira, feminista, pioneira em educação de enfermagem e ativista social - escreveu em 1907 o primeiro livro sobre história da enfermagem, obra em quatro volumes, cujo paradoxal título é A short history of nursing (Dingwall, Rafferty, Webster, 1988; White, 1978; Roberts, 1956). Textos acerca de sua história e de seus feitos começam a aparecer no ano de seu falecimento, 1956, com o artigo "Nurse, feminist, internationalist", publicado em um dos mais importantes periódicos americanos da época, o American Journal of Nursing. Em 2003 o último artigo proclama para seus pares a importância da enfermagem de saúde pública e suas pioneiras - "Public health nursing pioneer: Jane Elizabeth Hitchcock 1863-1939". Embora sem focalizar Lavinia Dock, enfatiza a importância de seu papel na formação das enfermeiras de saúde pública que a sucederam, incluindo a que consta do título.

A publicação das biografias brasileiras percorreu caminho diferente das biografias norteamericanas. O primeiro ponto a destacar é o fato de a maioria das biografadas ter sido contemplada com apenas um artigo retratando sua trajetória profissional e pessoal; são exceções Edith Magalhães Fraenckel, cuja história e importância é divulgada em três textos (1986, 1998, 2006), e as que mereceram dois artigos: Haydée Guanais Dourado, Glete de Alcântara, Zaíra Cintra Vidal, Maria Rosa Sousa Pinheiro e Waleska Paixão.

O segundo ponto diz respeito ao período histórico das publicações brasileiras: a quase totalidade (25 artigos de trinta publicados) se concentrou a partir de 2000. Isso não acontece por acaso: coincide com a ampliação dos grupos de pesquisa em história da enfermagem naquela década, quando foram criados 23 grupos, influenciados pelo fortalecimento dessa linha de pesquisa nos programas de pós-graduação em enfermagem no país. Atualmente, registrados no Diretório de Pesquisas do CNPq, encontramos 34 grupos de pesquisa em enfermagem que incluem a expressão 'história da enfermagem' no seu nome ou em suas linhas de pesquisa, o que favorece e fortalece a produção de conhecimento nessa área.

A história da enfermagem brasileira enquanto campo de conhecimento de importância para a profissão ainda se encontra em processo de sedimentação e ampliação, ficando essa valorização evidenciada na produção científica acerca dos personagens que fizeram e vêm fazendo diferença nos modelos profissionais. A ampliação dos estudos de natureza sóciohistórica nos últimos anos vem criando possibilidades de 'reconstrução' dos saberes constituintes dos contextos históricos e culturais específicos (Padilha, Borenstein, 2006).

Anna Justina Nery foi considerada a primeira enfermeira brasileira, sendo-lhe outorgado o título de 'Mãe dos Brasileiros' por sua atuação na Guerra do Paraguai. Ao retornar à Bahia trazendo seis órfãos paraguaios, desapareceu do cenário profissional; ainda assim deu nome à primeira escola de enfermagem moderna no Brasil, criada em 1922 (Cardoso, Miranda, 1999). 
Edith Magalhães Fraenckel, Haydée Guanais Dourado, irmã Tereza Notarnicola, Glete de Alcântara, Zaíra Cintra Vidal, Rachel Haddock Lobo, irmã Mathilde de Nina, Olga Verderese, Maria Rosa Sousa Pinheiro, Ottilie Hammes e Hilda Anna Krisch tiveram enfatizado em suas biografias o papel que exerceram junto à Associação Brasileira de Enfermagem desde sua fundação, em 1926, até sua ampliação nos demais estados da federação. As enfermeiras citadas foram essenciais na disseminação das escolas de enfermagem em todo país.

Clarice Della Torre Ferrarini, Maria A. Minzoni, Anna Nava, Grasiela Barroso, Eloita Pereira Neves, Alice Michaud, Waleska Paixão, Flora Costa Marques, Wilson Kraemer de Paula e Liselotte Hoeschl Ornellas também foram biografados por sua importância na criação de escolas de enfermagem e no desenvolvimento de área específica de conhecimento.

Wanda de Aguiar Horta é considerada a primeira teórica brasileira da enfermagem, e seu livro intitulado Processo de enfermagem (1979) é utilizado até os dias de hoje nas escolas de graduação. Nele encontra-se a apresentação da 'teoria das necessidades humanas', orientada pelas ideias do psicólogo norte-americano Abraham Maslow (1908-1970). Também criou o primeiro conceito formal brasileiro de enfermagem: "Enfermagem é a ciência e a arte de assistir o ser humano no atendimento de suas necessidades básicas, de torná-lo independente desta assistência através da educação; de recuperar, manter e promover sua saúde, contando para isso com a colaboração de outros grupos profissionais" (Horta, 1979, p.22).

Não há como negar que os significados da profissão de enfermagem influenciam sobremaneira a forma como o próprio conceito de enfermagem é compreendido. Isso pode ser exemplificado pelo discurso de Ethel Parsons (Carvalho, 1976) ${ }^{5}$ que, ao criar o Serviço de Enfermeiras do Departamento Nacional de Saúde Pública no Brasil em 1922, hesitou em utilizar a palavra enfermeira em português devido a sua conotação negativa na época, preferindo nurse, em inglês, a fim de diferenciar as profissionais formadas sob sua orientação das demais existentes na sociedade brasileira. ${ }^{6}$ O termo, porém, não teve aderência, prevalecendo formas como 'enfermeira de alto padrão, 'de saúde pública' ou simplesmente 'diplomada' para indicar a nova categoria que se criava (Alcântara, 1963). ${ }^{7}$

Após a diplomação da turma pioneira da Escola de Enfermeiras do Departamento Nacional de Saúde Pública, posteriormente Escola Anna Nery, em 1925, entendia-se que "a ideia da criação de uma associação de ex-alunas era conveniente tanto à Escola, que manteria um certo controle sobre o conjunto das enfermeiras por ela diplomadas, como as exalunas que ao seu diploma agregariam o capital cultural referente às credenciais de membro de uma associação profissional ligada a uma instituição de prestígio como a Escola Anna Nery" (Barreira, Sauthier, Baptista, 2001, p.157).

Edith Magalhães Fraenckel e Rachel Haddock Lobo, fundadoras da associação, também tiveram importância vital na criação, em 1932, do primeiro periódico de enfermagem, a revista Anais de Enfermagem, atual Revista Brasileira de Enfermagem, complementando o tripé necessário a uma profissão para ser reconhecida, ou seja, uma escola, uma associação e uma revista. Em termos de biografias na enfermagem e controversas discussões, Florence Nightingale desponta em inúmeros artigos e livros escritos por enfermeiras e historiadores interessados em sua história e nos papéis social, político, educativo e histórico que desempenhou ao longo da vida. Alguns textos são traduzidos para outros idiomas, como, 
por exemplo, o da própria biografada Notes on nursing (Nightingale, 1898), que mereceu edição em português (1983): Notas sobre enfermagem: o que é e o que não é.

A vida de Florence Nightingale e seu papel enquanto criadora da enfermagem moderna - por ter fundado a primeira escola de enfermagem dirigida por uma enfermeira e que tinha um currículo que atendia à prática da enfermagem, em 1860, junto ao Hospital Saint Thomas, em Londres -, são bastante enfatizados. Seu nome é destacado também como o de uma mulher que dedicou a vida ao cuidado do outro e à profissionalização da enfermagem, representada pela dama da lâmpada, incansável missionária (Paixão, 1979; Lunardi, 1998; Oguisso, 2006). ${ }^{8} \mathrm{Na}$ Inglaterra sua história é repassada às crianças como a de uma das grandes heroínas inglesas, além de ser considerada uma das cem personalidades que mudaram o mundo (Baly, 1998; Smith, 1982).

Florence Nightingale (1983, p.12) afirma que utilizou "a palavra enfermagem por falta de outra melhor"; entendia que a profissão era uma arte e, como tal, deveria apresentar qualidades que tornassem possível a assistência em enfermagem. Em Notas sobre enfermagem se refere ao que a enfermagem 'deve ser', apresentando aspectos que podem ser facilmente correlacionados com o que se espera de uma mulher: “Toda enfermeira deve ser uma pessoa com quem se pode contar, isto é, capaz de ser uma enfermeira 'de confiança' ... Deve ser estritamente sóbria, honesta e, mais do que isso, uma mulher religiosa e devotada. Deve ser uma observadora segura, direta e rápida, e uma mulher de sentimentos delicados e modestos" (p.164).

Por outro lado, Nightingale também é representada como pessoa perversa, capaz de manipulação maquiavélica para manter seu próprio poder e se autoengrandecer (Bullough, Bullough, Stanton, 1990; D’Antonio, 1995; Calabria, Macrae, 1994; Rosenberg, 1989). Outra contradição entre os autores que discutem a atuação de Florence Nightingale diz respeito a sua atuação como feminista, especialmente diante da resistência de sua família perante sua decisão de tornar-se enfermeira e por sua rejeição às expectativas da sociedade vitoriana para uma mulher de sua condição social (Hektor, 1994; Selanders, 1998; Malpas, 2006; Holliday, Parker, 1997).

Outros autores como Monteiro (1984) e Williams (2008) discordam dessa ideia e apresentam algumas atitudes de Florence Nightingale contrárias à postura feminista, tais como sua oposição à entrada de mulheres na profissão médica e sua recusa a participar do movimento pelo voto feminino e ampliação dos direitos das mulheres na sociedade inglesa (The New Women's Suffrage Society).

Ela compreendia a enfermagem como vocação e profissão, e os dois aspectos deviam estar unidos; a seu ver, qualquer mulher poderia vir a ser uma boa enfermeira. O sentimento de religiosidade marcou parcialmente o ideário da enfermagem, sobretudo quando a ele se aliaram qualidades desejadas tanto para religiosas quanto para enfermeiras, como "obediência, respeito à hierarquia, humildade, entre outras" (Baldwin, 1995; Clarke, 2006; Nelson, 2001).

De nosso ponto de vista seria enganoso atribuir somente às ideias de Florence Nightingale o movimento para criação e desenvolvimento da enfermagem moderna. Ela aprendeu enfermagem com as diaconisas de Kaiserwert, na Alemanha, e também com as irmãs de caridade de São Vicente de Paulo na França, vestindo-se, aliás, com o hábito das irmãs 
para, segundo declarou, delas absorver o espírito de cuidado e religião. Poderíamos dizer que, por meio da Escola e de tudo que escreveu sobre a forma de cuidar do outro, Florence Nightingale deu significado aos silêncios que havia na prática de enfermagem, até então envolta em regulamentos e correspondências internas, executados apenas por aquelas que faziam parte das associações específicas, na maioria das vezes religiosas, com espírito vocacional para servir o outro por amor a Deus, e não com o espírito e o desejo de construir uma profissão (Padilha, 1998).

O momento em que Florence Nightingale cria a profissão de enfermagem na Inglaterra coincide com as transformações evidenciadas por Michel Foucault no ambiente hospitalar, estabelecendo o vínculo entre os saberes da enfermagem e do médico numa situação de subordinação, considerando que até o século XVIII as irmãs de caridade dominavam o espaço hospitalar. Quando o médico percebe que o hospital é um campo de saber, e de consequente poder, assume esse espaço, e as irmãs de caridade o cedem passivamente, embora continuassem assegurando-o através do poder silencioso do cuidar e do domínio do ambiente e das chaves (Padilha, 1998). Com seus conhecimentos e a crença de que a enfermagem poderia ser uma profissão reconhecida, valorizada e exercida por mulheres de várias classes sociais, Nightingale propõe a retomada e a coletivização desse espaço.

Independentemente das críticas ou elogios, o nome de Florence Nightingale e os atributos recomendáveis a uma enfermeira confiável, como honestidade, sobriedade, religiosidade e devoção, habilidade de observação minuciosa, fidelidade e delicadeza, cruzaram os mares e se estabeleceram como o modelo de enfermeira a ser seguido para a criação dos primeiros cursos de enfermagem nos países que adotaram o sistema nightingaleano. As enfermeiras que frequentavam o curso de enfermagem eram consideradas enfermeiras-padrão, implicando o significado da expressão a presença de todos aqueles atributos.

Muitas enfermeiras, além de estabelecer uma nova profissão, transformaram o sistema de cuidado da saúde em seus países. A escrita das suas histórias permite a divulgação de seu trabalho e constitui fonte de informação para estudantes e interessados em história da enfermagem. Elas colocam em evidência a prática da profissão e sua importância para a sociedade. Associando os aspectos público e privado, as histórias de vida das enfermeiras recuperam a dimensão humana, muitas vezes negligenciada em biografias científicas (Roberts, 2002).

\section{Considerações finais}

As citações das histórias de vida dessas enfermeiras têm a função implícita de impregnar o espírito das estudantes e profissionais com o que se espera de uma enfermeira. Esses pontos contribuem para forjar sua identidade profissional, pois os modelos fornecem também os valores da profissão. Um ponto a discutir é: o uso didático dessas histórias de vida têm atingido esse objetivo em larga escala? Ou alcançam apenas enfermeiras historiadoras e/ou interessadas em história? Postular a instrumentalidade educativa da biografia coloca-nos em face de alguns problemas, tanto de natureza geral, relativos à biografia em si, quanto específicos, relacionados com sua abordagem na relação com a educação. A biografia trata do individual, da trajetória de uma vida específica, concreta. Quando 
empregada na educação, vincula as histórias dos indivíduos estudados com o coletivo dos conhecimentos, normas, valores, etc., isto é, com a instância coletiva.

Do ponto de vista epistemológico, como tratar da generalização? Como descobrir a interseção entre a trajetória individual e a da sociedade na qual essa trajetória é percorrida? Das respostas a essas questões dependerá a validade do uso da biografia como exemplo educativo, seja na disciplina de história da enfermagem seja em uma disciplina clínica. Trata-se de utilizar o individual em benefício do coletivo, de fazer com que as experiências, vivências e realizações de um indivíduo sejam apropriadas pela educação, tanto em seu âmbito formal e sistemático (a escola) quanto no sentido educativo mais amplo. Pretendese que a leitura das biografias influenciem as alunas com os exemplos que contêm, fornecendo subsídios para a construção da identidade profissional das enfermeiras.

Este estudo pretendeu abordar questões sobre a construção da identidade do profissional de enfermagem. Optamos por discutir o uso das biografias por entender que elas são essenciais para atingir essa finalidade. Também tivemos a intenção de sair do lugar-comum no qual autores repetem incansavelmente que "a história da enfermagem é importante para a construção da identidade profissional dos estudantes de enfermagem", mas raramente indicam os caminhos para se chegar lá.

Como afirmam Brown e D’Antonio (1990, p.319): “O ensino de nossa história não só concede a nossos estudantes e colegas amplo entendimento das origens de nossos atuais sucessos e dilemas, mas também proporciona aproximação analítica com as questões clínicas e profissionais complexas que contestam as explicações simplistas".

\section{NOTAS}

${ }^{1}$ A partir da década de 1960 o território do historiador estendeu-se a tudo o que é perceptível à observação. O historiador relê os documentos de seus predecessores com novos olhos, com nova interpretação e análise.

${ }^{2}$ Neste texto será utilizado o termo 'enfermeira', considerando-se que mais de $90 \%$ dos profissionais são do sexo feminino.

${ }^{3}$ O movimento da chamada 'história nova' se inicia na França com a criação, em 1929, da revista Annales d'histoire économique et sociale. A partir daí a compreensão de história se modifica e passa a valorizar não apenas o passado presente nos documentos, mas também na memória, nas vestes e em tudo o que pudesse ser rememorado. Alguns autores sobressaem nesse período, entre eles, Marc Bloch, François Simiand, Henri Hauser, Jacques Le Goff e Lucien Febvre.

${ }^{4}$ Essas autoras têm procurado indicar caminhos para o ensino da história da enfermagem nos cursos de graduação e pós-graduação em enfermagem no Brasil, Canadá e EUA.

${ }^{5}$ Ethel Parsons, do Serviço Internacional de Saúde da Fundação Rockefeller, que passou a chefiar a Missão Técnica de Cooperação para o Desenvolvimento da Enfermagem no Brasil em 1921 e criou o Serviço de Enfermeiras do Departamento Nacional de Saúde Pública em 1922, foi uma das organizadoras da Escola de Enfermagem Anna Nery, considerada a primeira escola moderna de enfermagem no Brasil.

${ }^{6}$ As pessoas chamadas de enfermeiras na época normalmente eram pobres, negras e escravas livres - o que não condizia com o modelo desejado - ou irmãs de caridade, que atuavam nos hospitais sem receber salário, como forma de servir a Deus.

${ }^{7}$ A expressão enfermeira de alto padrão continua a ser bastante utilizada para diferenciar a enfermeira graduada dos demais membros da equipe de enfermagem.

${ }^{8}$ No Brasil não há livro específico sobre Florence Nightingale; três a respeito da história da enfermagem em geral, entretanto, a incluem. Foram escritos por Waleska Paixão (1979), Valeria Lunardi (1998) e Taka Oguisso (2006). 


\section{REFERÊNCIAS}

ALCÂNTARA, Glete de.

A enfermagem moderna como categoria

profissional: obstáculos à sua expansão na

sociedade brasileira. Tese (cátedra) - Escola de

Enfermagem de Ribeirão Preto, Universidade de

São Paulo, Ribeirão Preto. 1963.

BALDWIN, Douglas.

Discipline, obedience and female support groups: Mona Wilson at the Johns Hopkins Hospital School of Nursing, 1915-1918. Bulletin of the History of Medicine, Baltimore, v.69, n.4, p.599-619. 1995.

BALY, Monica.

Florence Nightingale and the nursing legacy: building the foundation of modern nursing and midwifery. Philadelphia: Bainbridge Books. 1998.

BARREIRA, Ieda. A; SAUTHIER, Jussara; BAPTISTA, Suely S.

O movimento associativo das enfermeiras diplomadas brasileiras na 1ạ metade do século 20. Revista Brasileira de Enfermagem, Brasília, v.53, n.4, p.157-173. 2001.

BARREIRA, Ieda de Alencar et al.

Renovação no ensino e na pesquisa de história da enfermagem brasileira: a experiência da Escola de Enfermagem Anna Nery da Universidade Federal do Rio de Janeiro. Revista Enfermagem da UERJ, Rio de Janeiro, v.5, n.2, p.487-494. 1997.

BOCK, Ana.

A psicologia a caminho do novo século: identidade profissional e compromisso social. Estudos de Psicologia, Natal, v.4, n.2, p.78-89. 1999.

BROWN, Janie M.; D'ANTONIO, Patricia. Nursing history and scholarship: critical issues for the discipline. Journal of Professional Nursing, Philadelphia, v.6, n.6, p.319-348. 1990.

BUlLOUGH, Vern; BULlOUGH, Bonnie; STANTON, Marietta.

Florence Nightingale and her era. New York: Garland. 1990.

BURKE, Peter.

A Escola dos Annales (1929-1989). São Paulo: Editora da Unesp. 1991.

CALABRIA, Michael; MACRAE, Janet. Suggestions for thought by Florence Nightingale: selections and commentaries. Philadelphia: University of Pensylvania Press. 1994.

CARDOSO, Maria Manuela Vila Nova; MIRANDA, Cristina Maria Loyola.

Anna Justina Ferreira Nery: um marco na história da enfermagem brasileira. Revista Brasileira de Enfermagem, Brasília, v.52, n.3, p.339-348. 1999.

CARVALHO, Amalia.

Associação Brasileira de Enfermagem: 1926-1976. Brasília: Associação Brasileira de Enfermagem. 1976.

CERTEAU, Michel de.

A escrita da história. Rio de Janeiro: Forense Universitária. 1982.

CLARKE, L.

So what exactly is a nurse?. Journal of Psychiatric and Mental Health Nursing, Oxford, v.13, n.4, p.388-394. 2006.

COLL, M. et al.

Nursing care according to Virginia Henderson in the at home care field. Revista de Enfermería, Barcelona, v.30, n.3, p.53-56. 2007.

CORSINI, Leonora.

Repensando a identidade no contexto das migrações. Psicologia \& Sociedade, Florianópolis, v.18 n.3, p.23-33. 2006.

D’ANTONIO, Patricia.

Florence Nightingale by herself. Bulletin of the History of Medicine, Baltimore, v.69, n.2, p.278-287. 1995.

DINGWALL, Robert; RAFFERTY, Anne Marie; WEBSTER, Charles.

An introduction to the social history of nursing. London: Routledge. 1988.

EDER, Klaus.

Identidades coletivas e mobilização de identidades. Revista Brasileira de Ciências Sociais, São Paulo, v.18, n.53, p.340-356. 2003.

EISENSTADT, Shamuel N.

The construction of collective identities: some analytical and comparative indications. European Journal of Social Theory, Brighton, v.1, n.2, p.229-254. 1998.

HALAMAMDARIS, V.J.

Remembering Lillian Wald. Caring, Washington, v.28, n.3, p.64. 2009.

HAWKINS, J.W.; WATSON, J.C.

Public health nursing pioneer: Jane Elizabeth Hitchcock, 1863-1939. Public Health Nursing, Cambridge, v.20, n.3, p.167-176. 2003.

HEKTOR, Lynne M.

Florence Nightingale and the Women's Movement: friend or foe?. Nursing Inquiry, Carlton, v.1, n.1, p.38-45. 1994. 
HOBSBAWN, Eric; RANGER, Terence. The invention of tradition. Cambridge: Cambridge University Press. 1992.

HOLLIDAY, Mary; PARKER, David. Florence Nightingale, feminism and nursing. Journal of Advanced Nursing, Oxford, v.26, n.3, p.483-488. 1997.

HORTA, Wanda de Aguiar.

Processo de enfermagem. São Paulo: Editora Pedagógica e Universitária. 1979.

KIRBY, Stephanie.

Teaching nursing history: the Redwood experience. Nurse Education Today, Edinburgh, v.18, n.4, p.310-316. 1998.

LAIT, Margareth.

The place of nursing history in an undergraduate curriculum. Nurse Education Today, Edinburgh, v.20, n.5, p.325-336, 395-400. 2000 .

LE GOFF, Jacques.

A história nova. São Paulo: Martins Fontes. 1990.

LUDKE, Menga; BOING, Luiz Alberto. Caminhos da profissão e da profissionalidade docentes. Educação \& Sociedade, Campinas, v.25, n.89, p.1159-1180. 2004.

LUNARDI, Valeria Lerch.

História da enfermagem: rupturas e

continuidades. Pelotas: Ed.UFPel. 1998.

MALPAS, Peter.

Florence Nightingale: appreciating our legacy, envisioning our future. Gastroenterology Nursing, Baltimore, v.29, n.6, p.447-452. 2006.

MONTEIRO, Luiz.

On separate roads: Florence Nightingale and Elizabeth Blackwell. Signs, Pittsburgh, v.9, n.3, p.520-533. 1984.

\section{NELSON, Sioban.}

Say little, do much: nineteenth-century nursing and hospital foundation by religious women in the New World. Pennsylvania: University of Pennsylvania Press. 2001.

NIGHTINGALE, Florence.

Notas sobre enfermagem. São Paulo: Cortez. 1983.

NIGHTINGALE, Florence.

Notes on nursing: what it is, and what it is not. London: Harrison and Sons. 1.ed.,1860. 1898.

OGUISSO, Taka.

Trajetória histórica e legal da enfermagem. 2.ed. São Paulo: Manole. 2006.

OLIVEIRA, Roberto.

Os (des)caminhos da identidade. Revista
Brasileira de Ciências Sociais, São Paulo, v.15, n.42, p.7-21. 2000.

PADILHA, Maria Itayra Coelho de Souza. O ensino de história da enfermagem nos cursos de graduação de Santa Catarina. Trabalho, Educação e Saúde, Rio de Janeiro, v.4, n. 2, p.325-336. 2006.

PADILHA, Maria Itayra Coelho de Souza. A mística do silêncio: a enfermagem na Santa Casa de Misericórdia do Rio de Janeiro no século XIX. Pelotas: Ed.UFPel. 1998.

PADILHA, Maria Itayra Coelho de Souza; BORENSTEIN, Miriam Susskind.

História da enfermagem: ensino, pesquisa e interdisciplinaridade: Escola Anna Nery. Revista de Enfermagem, Rio de Janeiro, v.10, p.532-538. 2006.

PADILHA, Maria Itayra; NELSON, Sioban. Networks of identity: the potential of biographical studies for teaching nursing identity. Nursing History Review, Philadelphia, v.19, p.183-193. 2011.

PADILHA, Maria Itayra; NELSON, Sioban. Teaching nursing history: the Santa CatarinaBrazil experience. Nursing Inquiry, Carlton, v.16, p.171-180. 2009.

PAIXÃO, Waleska.

História da enfermagem. Rio de Janeiro: Júlio C. Reis Livraria. 1.ed.,1951. 1979.

PEREIRA, Fernanda; PEREIRA NETO, André. O psicólogo no Brasil: notas sobre seu processo de profissionalização. Psicologia em Estudo, Maringá, v.8, n.2, p.19-27. 2003.

POPIEL, E.S.

Lillian Wald, community nurse: try to remember our heritage. Colorado Nurse, Denver, v.68, n.1, p.3-5. 1968.

ROBERTS, Brian.

Biographical research: understanding social research. Buckingham: Open University Press. 2002.

ROBERTS, Mary May.

Lavinia Lloyd Dock: nurse, feminist,

internationalist. American Journal of Nursing, New York, v.56, n.2, p.176-179. 1956.

ROSENBERG, Charles.

Florence Nightingale on hospital reform. New York: Garland. 1989.

SANTOS, Miriam.

Sobre a autonomia das novas identidades coletivas: alguns problemas teóricos. Revista Brasileira de Ciências Sociais, São Paulo, v.13, n.38, p.46-56. 1998. 
Maria Itayra Padilha, Sioban Nelson, Miriam Susskind Borenstein

SELANDERS, Louise.

Florence Nightingale: the evolution and social impact of feminist values in nursing. Journal of Holistic Nursing, Springfield, v.16, n.2, p.227243. 1998.

SMITH, Francis.

Florence Nightingale: reputation and power. London: Croom Helm. 1982.

STRATH, Bo.

A European identity: to the historical limits of a concept. European Journal of Social Theory,

Brighton, v.5, n.4, p.387-401. 2002.
WHITE, Rosemary.

Social change and development of the nursing profession. London: Anchor Press. 1978.

WILLIAMS, K.

Reappraising Miss Nightingale. British Medical Journal, London, v.12, n.16, p.337-389. 2008.

YUMAKI, Masu.

Miss Virginia Henderson and I. Kango, Tokyo, v.28, n.8, p.56-59. 1976. 